\title{
New records of flowering plants of the flora of Myanmar collected from Natma Taung National Park (Chin State)
}

\author{
Dae-Hyun Kang, Shein Man Ling ${ }^{1}$, Young-Dong Kim and Homervergel G. Ong* \\ Department of Life Science, Hallym University, Chuncheon 24252, Korea \\ ${ }^{1}$ NTNP Office, Forest Department (MONREC/MoECAF), Kampetlet 03082, Chin State, Myanmar
}

(Received 8 September 2017; Revised 15 September 2017; Accepted 20 September 2017)

\begin{abstract}
The last four years of joint botanical collections by the governments of Myanmar and South Korea in Natma Taung National Park and adjacent areas in the Chin State of Myanmar have revealed the presence of 20 naturally occurring species of angiosperms new to the flora of Myanmar. Plants not previously recorded include species originally considered to be only found in neighboring mega-diverse countries. Examples (e.g., for India) include Boehmeria manipurensis Friis \& Wilmot-Dear (Urticaceae), Trigonotis hookeri Benth. ex C. B. Clarke (Boraginaceae) and Mycetia radiciflora (C. B. Clarke) Airy Shaw (Rubiaceae); those for China include Microtoena delavayi Prain (Lamiaceae), Pimpinella kingdon-wardii $\mathrm{H}$. Wolff (Apiaceae) and Senecio diversipinnus Y. Ling (Asteraceae). The data presented in this report are expected to be useful sources for phytogeographical studies of these species.
\end{abstract}

Keywords: Flowering plants, Myanmar, Natma Taung National Park, new records

Myanmar is the second largest country in Southeast Asia with an area of $678,500 \mathrm{~km}^{2}$. It occupies an important biogeographical position as it is located south of the Himalayan region predominantly bordered by regions and provinces of mega-diverse countries India (on the northwest) and China (on the northeast), all belonging to the eastern Asiatic floristic region. The country is also bordered by Thailand and Laos (on the south and southeast), making it as well, a component of the Indochina floristic region. Myanmar vegetation spans tropical evergreen, mixed deciduous, savanna and alpine types supporting a wealth of plant diversity which constitutes a significant component to the Indo-Burma biodiversity hotspot (Van Dijk et al., 2004; Tanaka, 2005).

Botanical exploration in the former Burma began in the late 19th century when it was still under the British colonial rule. The earliest works were those of Kurz (1877a, 1877b) and Hooker (1872, 1879, 1882, 1885, 1890, 1894, 1897). These were soon followed by plant enumerations by Lace (1912), Rodger (1922), Hundley and Chit (1961), and Hundley (1987), which were all later compiled by Kress et al. (2003) in a checklist of about 11,800 species of Myanmar spermatophytes.
The high biodiversity and abundance of natural resources in Myanmar are not only attributed to the country's geography, but also to its long economic and political isolation. Despite the country's rich plant diversity, however, very few new botanical collections have been made during the last halfcentury (Kress et al., 2003). Frodin (2001) hinted that the country perhaps had the smallest proportion of its flora collected in all of tropical Asia. Hence, much of its flora still remains to be learned, as well as of its floristic relations with neighboring countries in Asia (Frodin, 2001; Tanaka, 2005).

After the much needed government reforms in the past several years, the interest for systematic floristic investigations have finally been revived (e.g. Tanaka et al., 2006; Lee et al., 2014; Fujikawa et al., 2015; Tanaka et al., 2015). In 2011, the Forest Department of the Ministry of Natural Resources and Environmental Conservation (MONREC), Union of Myanmar and the National Institute of Biological Resources (NIBR) of the Ministry of Environment, Republic of Korea signed a memorandum of understanding to conduct collaborative floristic investigations in Natma Taung National Park (NTNP). Fig. 1 presents the research area.

\footnotetext{
*Author for correspondence: homervergelong@gmail.com
} 
NTNP is one of Myanmar's 43 existing PAs (Beffasti and Galanti, 2011), and is located between $20^{\circ} 45^{\prime}-22^{\circ} 00^{\prime} \mathrm{N}$ and $93^{\circ} 15^{\prime}-94^{\circ} 15^{\prime} \mathrm{E}$ in the country's northwestern region Chin State. The climate in this region is characterized by long rainywet season (May to early December) and relatively short cool-

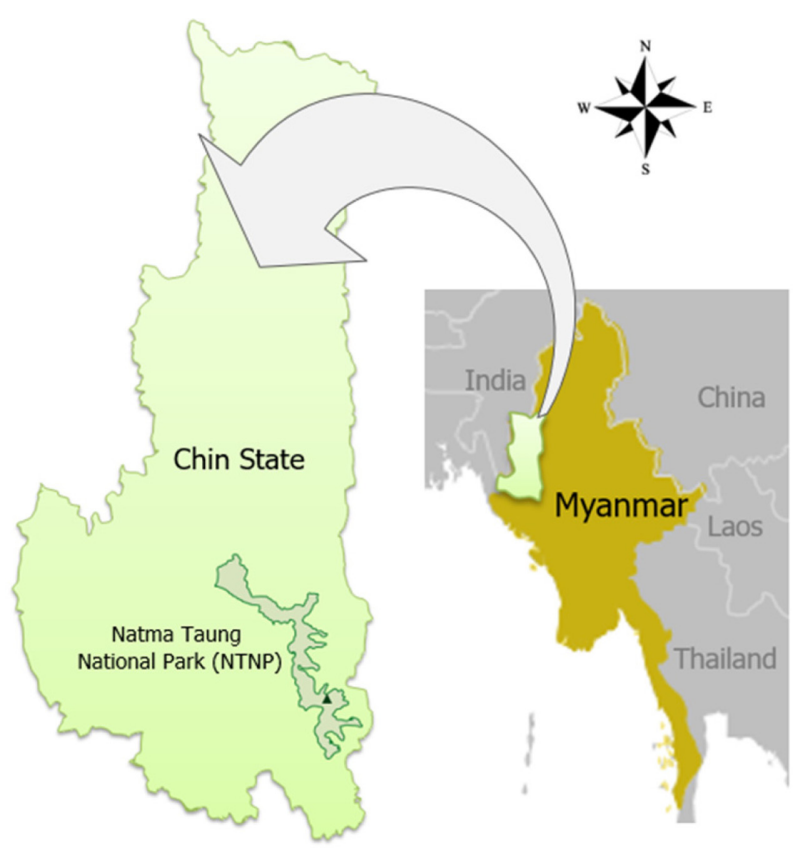

Fig. 1. Natma Taung National Park (NTNP) in Chin State, northwestern Myanmar. dry (mid-December to February) and hot-dry (March to April) seasons. Geopolitically, NTNP covers an area of $722.6 \mathrm{~km}^{2}$ within three townships (i.e., Kampetlet, Mindat, and Matupi) in southern Chin State. The general vegetation is made up of temperate and subtropical forests elevating to the montane meadows of the 3,053 meter-high summit of Mt. Natma Taung, which is recognized as the highest peak in the ruggedly mountainous region called the Chin Hills (Fujikawa et al., 2008). This region is bordered by the hill tracts of southwestern Bangladesh and the highland states of northeast India rising up in elevation to the Himalayas.

Due to the combined effects of its climate, geographic position and elevation, as well as human activities, NTNP and its adjacent areas have been reported to have a well-preserved biota characterized by a range of forest communities and high plant species endemism (Mill, 1995; Fujikawa et al., 2008). This paper reports the new records of flowering plants for the flora of Myanmar as a result of our years of investigations in NTNP and its neighboring areas in Chin State.

\section{Materials and Methods}

A total of nine expeditions in NTNP and its surrounding areas were conducted during different seasons from February 2013 to November 2016, each year with at least two field collections. Specimens were identified and were first checked in Kress et al. (2003) and other recently published local

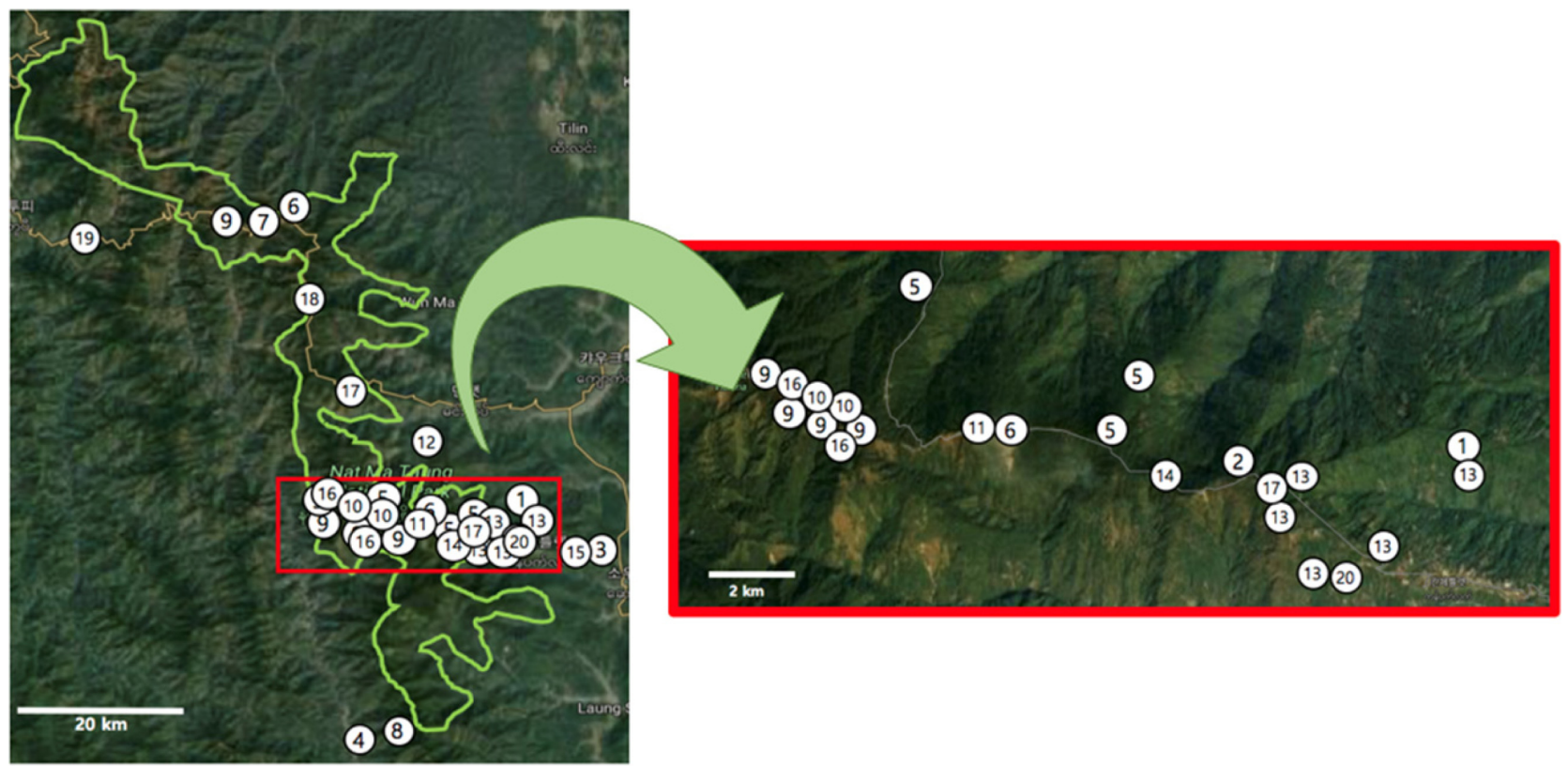

Fig. 2. Distribution of newly recorded Myanmar flowering plants in Natma Taung National Park (NTNP) and adjacent areas in Chin State. Numbers indicate collected species which are shown in the main list of new records. 
botanical literatures for their distribution in Myanmar (e.g. Tanaka et al., 2006; Lee et al., 2014; Fujikawa et al., 2015; Tanaka et al., 2015; Lee et al., 2016). Species not recorded in these references were further checked in different volumes of floristic records (e.g. Wu and Raven, 1994; Wu et al., 1994; Santisuk and Larsen, 2002; Van Welzen and Chayamarit, 2007) and most recent checklists (e.g. Cho et al., 2016) of adjacent countries. Voucher specimens were deposited at the Herbarium of Hallym University (HHU) and the National Institute of Biological Resources Herbarium (KB) in South Korea. The newly recorded species are arranged alphabetically by plant family, along with their voucher information. Taxonomic comments, notes on their distribution and type information are provided when necessary. The distribution of these species in NTNP and adjacent areas are mapped in Fig. 2.

\section{Results and Discussion}

Based on herbarium specimens from our plant collections made in the last four years, a total of 20 species representing new national records for Myanmar were documented. The species listed below are predominantly known to be distributed in the neighboring countries of Myanmar. These species belonging to 19 genera and 17 families, were found to be in their natural wild distribution hence, are more likely to represent under-recorded taxa. Species reportedly endemic to neighboring country India e.g., Boehmeria manipurensis Friis \& Wilmot-Dear (Urticaceae), Trigonotis hookeri Benth. ex C. B. Clarke (Boraginaceae) and Mycetia radiciflora (C. B. Clarke) Airy Shaw (Rubiaceae); and China e.g., Microtoena delavayi Prain (Lamiaceae), Pimpinella kingdon-wardii $\mathrm{H}$. Wolff (Apiaceae) and Senecio diversipinnus Y. Ling (Asteraceae) were also recorded in the research area, and are shown in Fig. 3. The data presented in this report are expected to be useful sources for phytogeographical studies of these species.

1. Dicliptera chinensis (L.) Juss. (Acanthaceae)

Specimens examined: Oak forest edges in Kampetlet, $21^{\circ} 13^{\prime} 10.6^{\prime \prime} \mathrm{N}, 94^{\circ} 03^{\prime} 42.8^{\prime \prime}$, elev. 1,510 m, 30 Mar 2014, Kim et al. MM-3340 (HHU, KB).

Type: China, Anon. s.n. (lectotype, LINN), seen as photo.

Distribution: Bangladesh, China, NE India, Vietnam (Wu et al., 1994).

2. Pimpinella kingdon-wardii H. Wolff (Apiaceae) (Fig. 3E) Specimens examined: NTNP area (Kampetlet) in montane oak and laurel forest, $21^{\circ} 12^{\prime} 52.9^{\prime \prime} \mathrm{N}, 94^{\circ} 00^{\prime} 28.9^{\prime \prime} \mathrm{E}$, elev.
2,369 m, 17 Aug 2016, Kim et al. MM-5603 (HHU).

Type: Tibet, Doker-la, Kingdon Ward 1100 (holotype) not seen.

Distribution: China (Wu et al., 2005).

3. Ceropegia juncea Roxb. (Apocynaceae)

Specimens examined: Along Kampetlet-Saw Road near Dipterocarpus and Shorea forest, $21^{\circ} 11^{\prime} 03.0^{\prime \prime} \mathrm{N}, 94^{\circ} 07^{\prime} 06.1^{\prime \prime} \mathrm{E}$, elev. 672 m, 22 Oct 2013, Kim et al. MM-2428 (HHU, KB).

Distribution: India, Sri Lanka (Murthy et al., 2012).

Note: This species has not been observed since its collection in 2013 after the road widening construction.

\section{Marsdenia lachnostoma Benth. (Apocynaceae)}

Specimens examined: Kyin Dway Village (Kampetlet) in secondary forest, 20 $57^{\prime} 59.7^{\prime \prime} \mathrm{N}, 93^{\circ} 50^{\prime} 42.1$ "E, elev. $694 \mathrm{~m}, 6$ Aug 2016, Kang et al. MM-5409 (HHU).

Type: China, Hong Kong, 1853, Wright s.n. (isotype, US), seen as photo.

Distribution: Cambodia, China, Laos, Thailand ( $\mathrm{Wu}$ and Raven, 1995).

\section{Schefflera hypoleucoides Harms (Araliaceae)}

Specimens examined: NTNP area (Kampetlet) in montane oak and laurel forest, $21^{\circ} 13^{\prime} 13.5^{\prime \prime} \mathrm{N}, 93^{\circ} 58^{\prime} 54.3^{\prime \prime} \mathrm{E}$, elev. 2,466 m, 23 Mar 2014, Kim et al. MM-3042 (HHU, KB); NTNP area along Kampetlet-Mindat Road on montane oak and laurel forest edges, $21^{\circ} 15^{\prime} 01.4^{\prime \prime} \mathrm{N}, 93^{\circ} 56^{\prime} 31.5^{\prime \prime} \mathrm{E}$, elev. 2,397 m, 18 Dec 2014, Kim et al. MM-3527 (HHU, KB); Pinus and oak forest, $21^{\circ} 14^{\prime} 05.4^{\prime \prime} \mathrm{N}, 93^{\circ} 59^{\prime} 29.0^{\prime \prime} \mathrm{E}$, elev. 2,182 m, 13 Jan 2016, Kim et al. MM-5095 (HHU, KB).

Type: China, Yuanchang 6,000 feet, Henry 13301 (isosyntype, A), seen as photo.

Distribution: China, Thailand, Vietnam (Wu et al., 2007c).

\section{Ixeridium gracile (DC.) Pak \& Kawano (Asteraceae)}

Specimens examined: NTNP area along Kampetlet-Mindat Road on laurel forest edges, $21^{\circ} 13^{\prime} 14.5^{\prime \prime} \mathrm{N}, 93^{\circ} 57^{\prime} 35.4 " \mathrm{E}$, elev. 2,572 m, 18 May 2015, Kim et al. MM-4033 (HHU, KB); NTNP area along Mindat-Matupi Road, 2136'56.3"N, 93'46'27.0"E, elev. 2,268 m, 23 May 2015, Kim et al. MM4295 (HHU, KB).

Type: Nepal, Wallich $3267 a$ (isosyntype, BM), seen as photo.

Distribution: Bhutan, China, NE India, Nepal (Wu et al., 2011).

7. Senecio diversipinnus Y. Ling (Asteraceae) (Fig. 3F) 

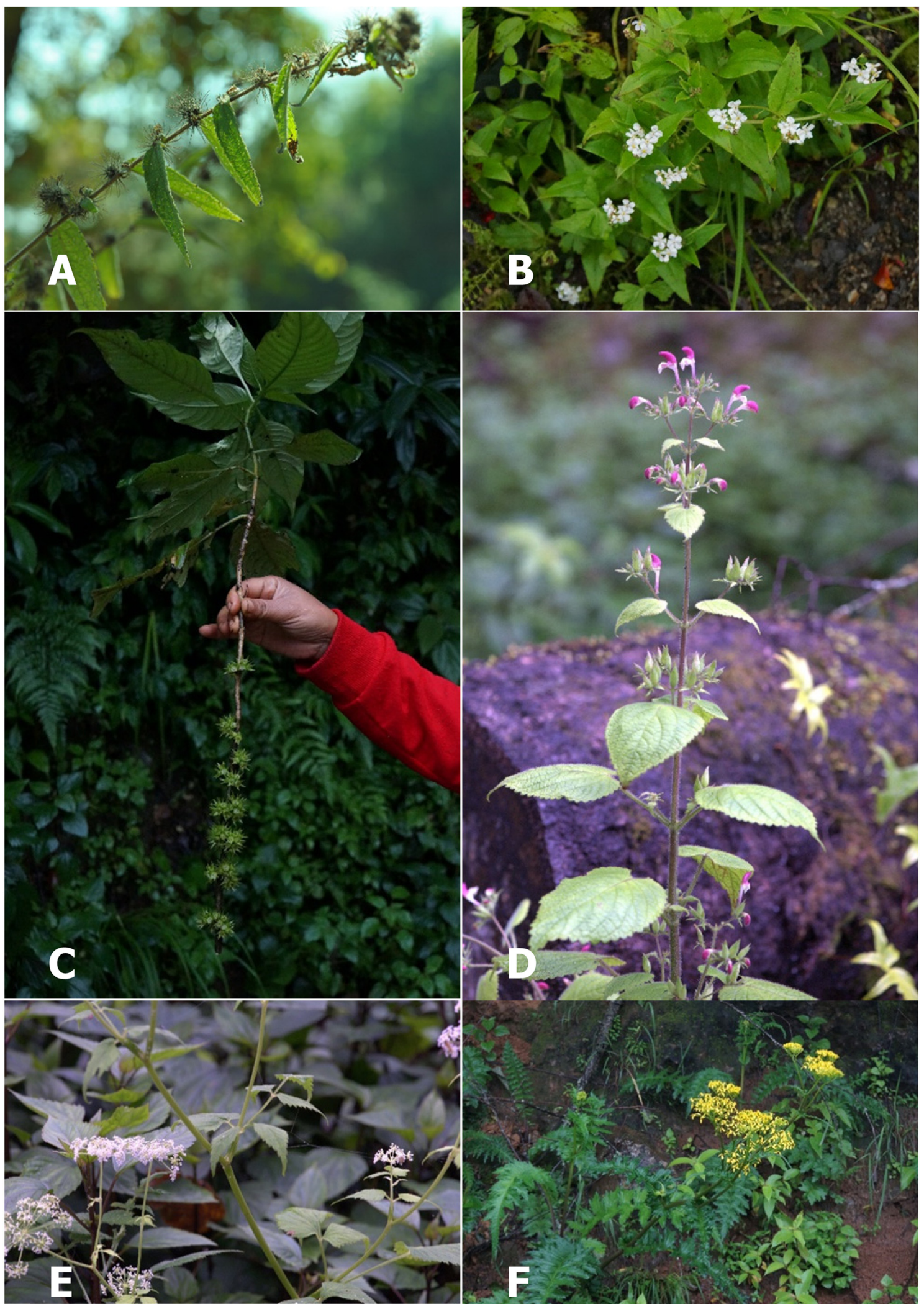

Fig. 3. Myanmar new records previously reported being distributed only in India and China. A-C. Reportedly India endemics. A. Boehmeria manipurensis. B. Trigonotis hookeri. C. Mycetia radiciflora. D-F. Reportedly China endemics. D. Microtoena delavayi. E. Pimpinella kingdon-wardii. F. Senecio diversipinnus. 
Specimens examined: NTNP area grassland near MindatMatupi Road, 21 $35^{\prime} 41.8^{\prime \prime} \mathrm{N}, 93^{\circ} 43^{\prime} 55.4^{\prime \prime} \mathrm{E}$, elev. 2,409 m, 25 Jul 2015. Kim et al. MM-4562 (HHU, KB).

Type: China, 20 Jul 1936, T. P. Wang 5136 (syntype, PE), seen as photo.

Distribution: China (Wu et al., 2011).

8. Begonia dioica Buch.-Ham. ex D. Don (Begoniaceae)

Specimens examined: Along road to Kyin Dway Village (Kampetlet) near spring drinking water source, 20 $59^{\prime} 13.9^{\prime \prime} \mathrm{N}$, 935' 19.2"E, elev. 1,940 m, 12 Aug 2016, Kang et al. MM5520 (HHU).

Type: Nepal, 1821, Wallich 3682 (holotype, K), seen as photo.

Distribution: Nepal (Don, 1825), India (Uddin, 2007), Bhutan (Grierson and Long, 1991).

9. Trigonotis hookeri Benth. ex C. B. Clarke (Boraginaceae) (Fig. 3B)

Specimens examined: NTNP area near summit of Mt. Natma Taung on oak and Rhododendron forest edges, $21^{\circ} 13^{\prime} 11.2^{\prime \prime} \mathrm{N}, 93^{\circ} 56^{\prime} 03.3^{\prime \prime} \mathrm{E}$, elev. 2,797 m, 21 Oct 2013, Kim et al. MM-2230 (HHU, KB); $21^{\circ} 13^{\prime} 17.4^{\prime \prime} \mathrm{N}, 93^{\circ} 55^{\prime} 32.4^{\prime \prime} \mathrm{E}$, elev. 2,969 m, 21 Oct 2013, Kim et al. MM-2331 (HHU, KB); $21^{\circ} 13^{\prime} 41.9^{\prime \prime} \mathrm{N}, 93^{\circ} 54^{\prime} 41.1^{\prime \prime}$, elev. 2,966 m, 24 Oct 2013, Kim et al. MM-2787 (HHU, KB); NTNP area grassland along Mindat-Matupi Road, 2135'53.6"N, 9341'52.1"E, elev. 2,395 m, 25 Jul 2015, Kim et al. MM-4527 (HHU, KB), Kim et al. MM-4540-1 (HHU, KB); NTNP area on Mt. Natma Taung peak meadows, $21^{\circ} 14^{\prime} 01.8^{\prime \prime} \mathrm{N}, 93^{\circ} 54^{\prime} 12.0^{\prime \prime} \mathrm{E}$, elev. 3,055 m, 29 Jul 2015, Kim et al. MM-4816 (HHU).

Type: India, Khasia, Hooker \& Thomson s.n. (type, BM), seen as photo.

Distribution: India (Hooker, 1885).

Note: This plant was found growing abundantly on peak meadows and open areas from ca. 2,400 m.

\section{Codonopsis viridis Wall. (Campanulaceae)}

Specimens examined: NTNP area near summit of Mt. Natma Taung on oak and Rhododendron forest edges, $21^{\circ} 13^{\prime} 41.9^{\prime \prime} \mathrm{N}, 93^{\circ} 54^{\prime} 41.1^{\prime \prime} \mathrm{E}$, elev. 2,966 m, 24 Oct 2013, Kim et al. MM-2771 (HHU, KB); 21 ${ }^{\circ} 13^{\prime} 42.1^{\prime \prime N}, 93^{\circ} 54^{\prime} 44.3^{\prime \prime} \mathrm{E}$, elev. 2,943 m, 17 Dec 2014, Kim et al. MM-3481 (HHU, KB).

Type: Nepal, Napaul Kamaan, Wallich 298 (type, L), seen as photo.

Distribution: Bhutan, China, N India, Nepal, Pakistan (Wu et al., 1994b).

Note: This plant is rare and was only observed near the summit of Mt. Natma Taung and not in other areas surveyed.

11. Gaultheria longibracteolata R. C. Fang (Ericaceae)

Specimens examined: NTNP area near Kampetlet-Mindat Road on laurel forest edges, $21^{\circ} 13^{\prime} 14^{\prime \prime} \mathrm{N}, 93^{\circ} 57^{\prime} 35^{\prime \prime} \mathrm{E}$, elev. 2,560 m, 3 Feb 2014, Ling S. M. SM-41 (HHU).

Type: China, S Yunnan: Luchun Xian, 1,700 m, 17 Sep 1973, D. D. Tao 68 (holotype, KUN; isotype, KUN), not seen.

Distribution: China, Thailand (Wu et al., 2005; Santisuk and Balslev, 2015).

12. Macaranga peltata (Roxb.) Müll. Arg. (Euphorbiaceae) Specimens examined: Kampetlet-Mindat area in oak forest, $21^{\circ} 19^{\prime} 39.5^{\prime \prime} \mathrm{N}, 93^{\circ} 56^{\prime} 07.5^{\prime \prime E}$, elev. 1,341 m, 24 Jul 2015, Kim et al. $M M-4502$ (HHU, KB).

Type: India, Roxburgh s.n. (type, BM), seen as photo.

Distribution: India, N Thailand, Sri Lanka (Whitmore, 1978; Van Welzen and Chayamarit, 2007).

Note: $M$. peltata can be distinguished from M. indica by its ovate bracteoles and fruit size twice as big as that of the latter.

\section{Ostodes katharinae Pax (Euphorbiaceae)}

Specimens examined: NTNP area (Kampetlet) in secondary forest, 21 ${ }^{\circ} 11^{\prime} 56.1^{\prime \prime} \mathrm{N}, 94^{\circ} 02^{\prime} 30.3^{\prime}$ 'E, elev. 1,702 m, 21 Feb 2013, Kim et al. MM-1561 (HHU); Pinus forest (Kampetlet), $21^{\circ} 12^{\prime} 12.4^{\prime \prime}$, 9401'25.8”'E, elev. 2,014 m, 20 Oct 2013, Kim et al. MM-1973 (HHU); Alnus forest (Kampetlet) near roads and shifting cultivation area, $21^{\circ} 12^{\prime} 38.1^{\prime \prime} \mathrm{N}, 94^{\circ} 01$ '25.4'E, elev. 2,123 m, 25 Mar 2014, Kim et al. MM-3143 (HHU); Oak forest (Kampetlet), 21 $1^{\circ} 12^{\prime} 55.6^{\prime \prime} \mathrm{N}, 94^{\circ} 03^{\prime} 33.6$ 'E, elev. 1,436 m, 30 Mar 2014, Kim et al. MM-3372 (HHU), Kim et al. MM-3375 (HHU); $21^{\circ} 11^{\prime} 35.8^{\prime \prime} \mathrm{N}, 94^{\circ} 01^{\prime} 46.0^{\prime \prime} \mathrm{E}$, elev. 1,596 m, 15 May 2015, Kim et al. MM-3918 (HHU).

Type: China, Yunnan Szemao, 1938, Henry 13062 (Syntype, $\mathrm{K})$, seen as photo.

Distribution: China, N Thailand (Wu et al., 2008b).

14. Microtoena delavayi Prain (Lamiaceae) (Fig. 3D)

Specimens examined: NTNP area (Kampetlet) on montane oak and laurel forest edges, $21^{\circ} 12^{\prime} 41.4^{\prime \prime} \mathrm{N}, 93^{\circ} 59^{\prime} 47.1^{\prime \prime} \mathrm{E}$, elev. 2,383 m, 17 Aug 2016, Kim et al. MM-5593 (HHU).

Type: China, Yunnan, les bois dans les gores de San Tchang Kiou (Ho Kin), 19 Aug 1886, Delavay 2463 (holotype, P), seen as photo.

Distribution: China (Wu and Raven, 1994).

Note: $M$. delavayi var. delavayi can be distinguished from $M$. delavayi var. amblyodon by its narrowly lanceolate calyx teeth, and from $M$. delavayi var. lutea and $M$. delavayi var. 
grandiflora by its longer corolla tube.

15. Syzygium globiflorum (Craib) Chantaran. \& J. Parn. (Myrtaceae)

Specimens examined: Kampetlet-Saw area in Dipterocarpus and Shorea forest, $21^{\circ} 11^{\prime} 07.3^{\prime \prime} \mathrm{N}, 94^{\circ} 06^{\prime} 40.9^{\prime \prime} \mathrm{E}$, elev. 767 m, 21 May 2015, Kim et al. MM-4198 (HHU, KB).

Type: Thailand, Doi Pa Kao, 7 May 1921, Kerr 5388 (isolectotype, K), seen as photo.

Distribution: China, Thailand (Santisuk and Larsen, 2002; Wu et al., 2007c).

16. Corydalis changuensis D. G. Long (Papaveraceae)

Specimens examined: NTNP area near Mt. Natma Taung summit in oak and Rhododendron forest, $21^{\circ} 13^{\prime} 17.4^{\prime \prime} \mathrm{N}$, 93'55'32.4"E, elev. 2,969 m, 21 Oct 2013, Kim et al. MM-2328 (HHU, KB); NTNP area on Mt. Natma Taung peak meadows, $21^{\circ} 13^{\prime} 59.5^{\prime \prime} \mathrm{N}, 93^{\circ} 54^{\prime} 19.2^{\prime \prime E}$, elev. 3,028 m, 29 Jul 2016, Kim et al. MM-4823 (HHU, KB).

Type: India, Changu, 9 Sep 1913, Cooper 845 (holotype, E), seen as photo.

Distribution: China, NE India (Wu et al., 2008a).

Note: $C$. changuensis was only observed near the summit of Mt. Natma Taung. It is distinguished from C. rubrisepala subsp. zhuangiana Lidén by its black-tipped inner petals.

\section{Rhamnus procumbens Edgew. (Rhamnaceae)}

Specimens examined: NTNP area near Mindat-Matupi Road in montane oak and laurel forest, $21^{\circ} 23^{\prime} 58.6^{\prime \prime} \mathrm{N}$, 9350'56.9"E, elev. 2,250 m, 22 May 2015, Kim et al. MM4267 (HHU, KB); NTNP area near Kampetlet-Mindat Road in montane oak and laurel forest, $21^{\circ} 12^{\prime} 40.2^{\prime \prime} \mathrm{N}, 94^{\circ} 01^{\prime} 08.4^{\prime \prime} \mathrm{E}$, elev. 2,282 m, 24 May 2015, Kim et al. MM-4404 (HHU, $\mathrm{KB})$.

Distribution: Bhutan, China, NW India, Nepal (Wu et al., 2007b).

Note: $R$. procumbens is generally distinguished from $R$. heterophylla Oliver by its bisexual flowers.

18. Neillia rubiflora D. Don (Rosaceae)

Specimens examined: NTNP area near Mindat-Matupi Road in montane oak and laurel forest, $21^{\circ} 30^{\prime} 57.6^{\prime \prime} \mathrm{N}$, 934' 15.0"E, elev. 2,563 m, 23 May 2015, Kim et al. MM4346 (HHU, KB).

Distribution: Bhutan, China, India, Nepal ( $\mathrm{Wu}$ et al., 2007a).

Type: Nepal, Wallich 697 (type, GZU), seen as photo.

Note: N. rubiflora was found growing together with $N$. thyrsiflora D. Don in the surveyed area, but can be easily distinguished by its raceme inflorescence.

19. Mycetia radiciflora (C. B. Clarke) Airy Shaw (Rubiaceae) (Fig. 3C)

Specimens examined: Near Mindat-Matupi Road, 21'34'22.9"N, 9331'36.1"E, elev. 1,300 m, 27 Jul 2015, Kim et al. $M M-4676$ (HHU, KB).

Type: India, Neechoogard. Naga Hills, 17 Oct 1885, Clarke 40847 (type, K), seen as photo.

Distribution: NE India (Airy Shaw, 1965).

20. Boehmeria manipurensis Friis \& Wilmot-Dear (Urticaceae) (Fig. 3A)

Specimens examined: Kampetlet area near small waterfall in secondary forest, $21^{\circ} 11^{\prime} 36.8^{\prime \prime} \mathrm{N}, 94^{\circ} 01^{\prime} 33.9^{\prime \prime} \mathrm{E}$, elev. 1,578 m, 16 Jan 2016, Kim et al. MM-5235 (HHU, KB).

Type: India, Irang Manipur, Nov 1907, Meebold 6253 (holotype, K), seen as photo.

Distribution: NE India (Wilmot-Dear et al., 2010).

\section{Acknowledgments}

This work was supported by grants from the National Institute of Biological Resources (NIBR), the Ministry of Environment (MOE) of the Republic of Korea (NIBR2016 04201). Our gratitude extends to the Ministry of Natural Resources and Environmental Conservation of Myanmar for the continuous guidance and support for this project.

\section{Literature Cited}

Airy Shaw, H. K. 1965. On a new species of the genus Silvianthus Hook. f., and on the family Carlemanniaceae. Kew Bulletin 19: $507-512$.

Beffasti, L. and V. Galanti. 2011. Myanmar Protected Areas: Context, Current Status and Challenges. Ancora Libri Press, Milan. Pp. 72-73.

Cho, S.-H., P. Chhang and Y.-D. Kim. 2016. A Checklist for the Seed Plants of Cambodia. National Institute of Biological Resources, Incheon, 272 pp.

Don, D. 1825. Prodromus Florae Nepalensis. London, 256 pp.

Frodin, D. G. 2001. Guide to Standard Floras of the World. 2nd ed. Cambridge University Press, Cambridge, 1100 pp.

Fujikawa, K., N. Kuroiwa, A. Maeda, S. Gale, S. G. Ngai and A. Din. 2008. A Guide to the Forests of Natma Taung: Natma Taung National Park, Myanmar. The Kochi Prefectural Makino Botanical Garden, Kochi, 30 pp. 
Fujikawa, K., P. Srisanga, S. Watthana, L. S. Mane, L. Shine, T. M. Soe and S. G. Ngai. 2015. 100 Wild Flowers of Natma Taung: Natma Taung National Park, Chin State, Myanmar. The Kochi Prefectural Makino Botanical Garden, Kochi, 76 pp.

Grierson, A. J. C. and D. G. Long. 1991. Flora of Bhutan. Vol. 2. Royal Botanic Garden Edinburgh, Edinburgh, 426 pp.

Hooker, J. D. 1872. Flora of British India. Vol. 1. Ranunculaceae to Polygaleae. L. Reeve \& Co., London, 740 pp.

Hooker, J. D. 1879. Flora of British India. Vol. 2. Sabiaceae to Cornaceae. L. Reeve \& Co., London, 792 pp.

Hooker, J. D. 1882. Flora of British India Vol. 3. Caprifoliaceae to Apocynaceae. L. Reeve \& Co., London, 712 pp.

Hooker, J. D. 1885. Flora of British India Vol. 4. Asclepiadeae to Amarantaceae. L. Reeve \& Co., London, 630 pp.

Hooker, J. D. 1890. Flora of British India. Vol. 5. Chenopodiaceae to Orchideae. L. Reeve \& Co., London, 255 pp.

Hooker, J. D. 1894. Flora of British India. Vol. 6. Orchideae to Cyperaceae. L. Reeve \& Co., London, 792 pp.

Hooker, J. D. 1897. Flora of British India. Vol. 7. Cyperaceae, Gramineae and General Index. L. Reeve \& Co., London, 842 pp.

Hundley, H. G. 1987. List of Trees, Shrubs, Herbs and Principal Climbers, etc. Recorded from Burma with Vernacular Names. 4th revised ed. Forest Department, Swe Daw Oo Press, Rangoon, $568 \mathrm{pp}$.

Hundley, H. G. and K. K. Chit. 1961. List of Trees, Shrubs, Herbs and Principal Climbers, etc. Recorded from Burma with Vernacular Names. 3rd ed. Supdt. Govt. Printing and Staty., Rangoon, $532 \mathrm{pp}$.

Kress, W. J., R. A. De Filipps, E. Farr and D. Y. Y. Kyi. 2003. A checklist of the trees, shrubs, herbs, and climbers of Myanmar (revised from the original works by J. H. Lace, R. Rodger, H. G. Hundley, and U Chit Ko Ko on the "List of Trees, Shrubs, Herbs and Principal Climbers, etc. Recorded from Burma"). Contributions from the Unites States National Herbarium 45: 590 pp.

Kurz, S. 1877a. Forest Flora of British Burma. Vol. 1. Ranunculaceae to Cornaceae. Office of the Superintendent of Government Printing, Calcutta, 549 pp.

Kurz, S. 1877b. Forest Flora of British Burma Vol. 2 Caprifoliaceae to Filices. Office of the Superintendent of Government Printing, Calcutta, 613 pp.

Lace, J. H. 1912. List of Trees, Shrubs, Herbs and Principal Climbers, etc. Recorded from Burma. Forest Department, Rangoon, 394 pp.

Lee, W-S., N. Z. Htun, N. M. Lay, J-I. Choe, J-H. Jung, J-K. Park, J-Y. Park, W. N. Zarchi, E-Y. Choi, I-J., Choi, Y-K. Oh, J-L.
Lim, J-B. Choi, Y-D. Kim and S-H. Cho. 2014. Biodiversity of Mt. Popa, Myanmar. National Institute of Biological Resources, Incheon, 224 pp.

Lee, W-S., J-I. Choe, H-K. Kim, J-H. Jung, J-H. Jeon, J-S. Kim, JU. Kim, M-S. Jeong, J-K. Park, E-Y. Choi, W. N. Zarchi, S-Y. Kim, J-B. Choi, D-I. Cho, M-H. Kim, D-H. Kang, S-H. Cho, J-H. Lee, H. G. Ong, Y-I. Kim, B-Y. Kim, H-S. Park, S. M. Ling, Y-D. Kim, M-O. Moon, S-J. Pak, N-R. Yoon, T. Y. New and B-Y. Sun. 2016. Biodiversity of Natma Taung National Park, Myanmar. National Institute of Biological Resources (NIBR), Incheon, 288 pp.

Mill, R. R. 1995. Regional overview: Indian subcontinent. In Centres of Plant Diversity: A Guide to Strategy for their Conservation - Asia, Australia and the Pacific. Vol. 10. Davis, S. D., V. H. Heywood and A. C. Hamilton (eds.), World Wildlife Fund for Nature and IUCN Press, Cambridge. Pp. 62-135.

Murthy, K. S. R., R. Kondamudi, M. C. Reddy, S. Karuppusamy and T. Pullaiah. 2012. Check-list and conservation strategies of the genus Ceropegia in India. International Journal of Biodiversity and Conservation 4: 304-315.

Rodger, A. 1922. List of Trees, Shrubs, Herbs and Principal Climbers, etc. Recorded from Burma with Vernacular Names. 2nd ed. Forest Department, Rangoon, 532 pp.

Santisuk, T. and H. Balslev. 2015. Flora of Thailand. Vol. 13. Part 1. Achariaceae, Adoxaceae, Cannabaceae, Caprifoliaceae, Ericaceae, Salicaceae \& Ulmaceae. Royal Forest Department, Bangkok, $141 \mathrm{pp}$.

Santisuk, T. and K. Larsen. 2002. Flora of Thailand. Vol. 7. Part 4. Buddlejaceae, Hydrangaceae, Loranthaceae, Myrisicaceae, Myrtaceae, Saxifragaceae, Visaceae. Royal Forest Department, Bangkok, 285 pp.

Tanaka, N. 2005. Plant inventory research: contributions to the flora of Myanmar. Acta Phytotaxonomica et Geobotanica 56: $21-26$.

Tanaka, N., T. Koyama and J. Murata. 2006. The flowering plants of Mt. Popa, Central Myanmar. Bulletin of the Makino Botanical Garden 5: 1-102.

Tanaka, N., T. Yukawa, K. M. Htwe and J. Murata. 2015. An orchid checklist of Mt. Popa, Central Myanmar. Bulletin of the National Museum of Nature and Science, Series B (Botany), Tokyo 41: 69-89.

Uddin, A. 2007. Distribution and status of Indian Begonia L. species. Journal of Economic and Taxonomy Botany 31: 591597.

Van Dijk, P. P., A. W. Tordoff, J. Fellowes, M. Lau and M. Jinshuang. 2004. Indo-Burma. In Hotspots Revisited: Earth's Biologically Richest and Most Endangered Terrestrial Ecoregions. Mittermeier, R. A., P. Robles Gil, M. Hoffmann, J. Pilgrim, T. 
Brooks, C. G. Mittermeier, J. Lamoreaux and G. A. B. da Fonseca (eds.), CEMEX, Agrupación Sierra Madre. Pp. 323-330. Van Welzen P. C. and K. Chayamarit. 2007. Flora of Thailand. Vol. 8. Part 2. Euphorbiaceae. Royal Forest Department, Bangkok, 314 pp.

Whitmore, T. C. 1978. Studies in Macaranga VII. The genus in 'Greater India'. Gardens' Bulletin Singapore 16: 51-56.

Wilmot-Dear, C. M., I. Friis and Z. Thomas. 2010. New species in old world Boehmeria (Urticaceae). Edinburgh Journal of Botany 67: 431-450.

Wu, Z. Y. and P. H. Raven. 1994. Flora of China. Vol. 17. Verbenaceae to Solanaceae. Science Press, Beijing and Missouri Botanical Garden Press, St. Louis, MO, 342 pp.

Wu, Z. Y. and P. H. Raven. 1995. Flora of China. Vol. 16. Gentianaceae to Boraginaceae. Science Press, Beijing and Missouri Botanical Garden Press, St. Louis, MO, 479 pp.

Wu, Z. Y., P. H. Raven and D. Y. Hong. 1994. Flora of China. Vol. 19. Cucurbitaceae through Valerianaceae with Annonaceae and Berberidaceae. Science Press, Beijing and Missouri Botanical Garden Press, St. Louis, MO, 884 pp.

Wu, Z. Y., P. H. Raven and D. Y. Hong. 2005. Flora of China. Vol. 14. Apiaceae to Ericaceae. Science Press, Beijing and Mis- souri Botanical Garden Press, St. Louis, MO, 292 pp.

Wu, Z. Y., P. H. Raven and D. Y. Hong. 2007a. Flora of China. Vol. 9. Pittosporaceae to Connaraceae. Science Press, Beijing; Missouri Botanical Garden Press, St. Louis, MO, 496 pp.

Wu, Z. Y., P. H. Raven and D. Y. Hong. 2007b. Flora of China. Vol. 12. Hippocastanaceae to Theaceae. Science Press, Beijing and Missouri Botanical Garden Press, St. Louis, MO, 534 pp.

Wu, Z. Y., P. H. Raven and D. Y. Hong. 2007c. Flora of China. Vol. 13. Clusiaceae to Araliaceae. Science Press, Beijing and Missouri Botanical Garden Press, St. Louis, MO, 548 pp.

Wu, Z. Y., P. H. Raven and D. Y. Hong. 2008a. Flora of China. Vol. 7. Menispermaceae to Capparaceae. Science Press, Beijing and Missouri Botanical Garden Press, St. Louis, MO, 499 pp.

Wu, Z. Y., P. H. Raven and D. Y. Hong. 2008b. Flora of China. Vol. 11. Oxalidaceae to Aceraceae. Science Press, Beijing and Missouri Botanical Garden Press, St. Louis, MO, 622 pp.

Wu, Z. Y., P. H. Raven and D. Y. Hong. 2011. Flora of China. Vol. 20-21 Asteraceae. Science Press, Beijing and Missouri Botanical Garden Press, St. Louis, MO, 992 pp. 